\title{
A Tough Flexible Cellulose Nanofiber Air Cathode For ORR With Silver Nanoparticles and Carbon Nanotubes In Rechargeable Zinc Air Batteries
}

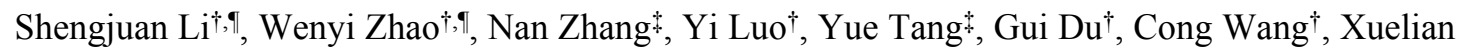
Zhang§, Lei Li*,†

†School of Materials Science and Engineering, University of Shanghai for Science and Technology, 516 Jungong Road, Shanghai 200093, P.R. China

ॠShanghai Tonnor Material Science Co., Ltd, Shanghai 200433, P.R. China

${ }^{\S}$ Department of Chemistry and Chemical Engineering, Hulunbuir University, Hailar 031108, P.R. China

*Corresponding author:

Lei Li (E-mail: $\underline{\text { lilei@usst.edu.cn; Tel: +86-21-55271656) }}$

"The first two authors contributed equally to this work. 
Typical SEM images of each membrane are shown in Fig. S1. It can be seen that the fibers three-dimensionally entangled with each other to form a porous structure. In addition, the loading of silver nanoparticles has no effect on the morphology of the composites.
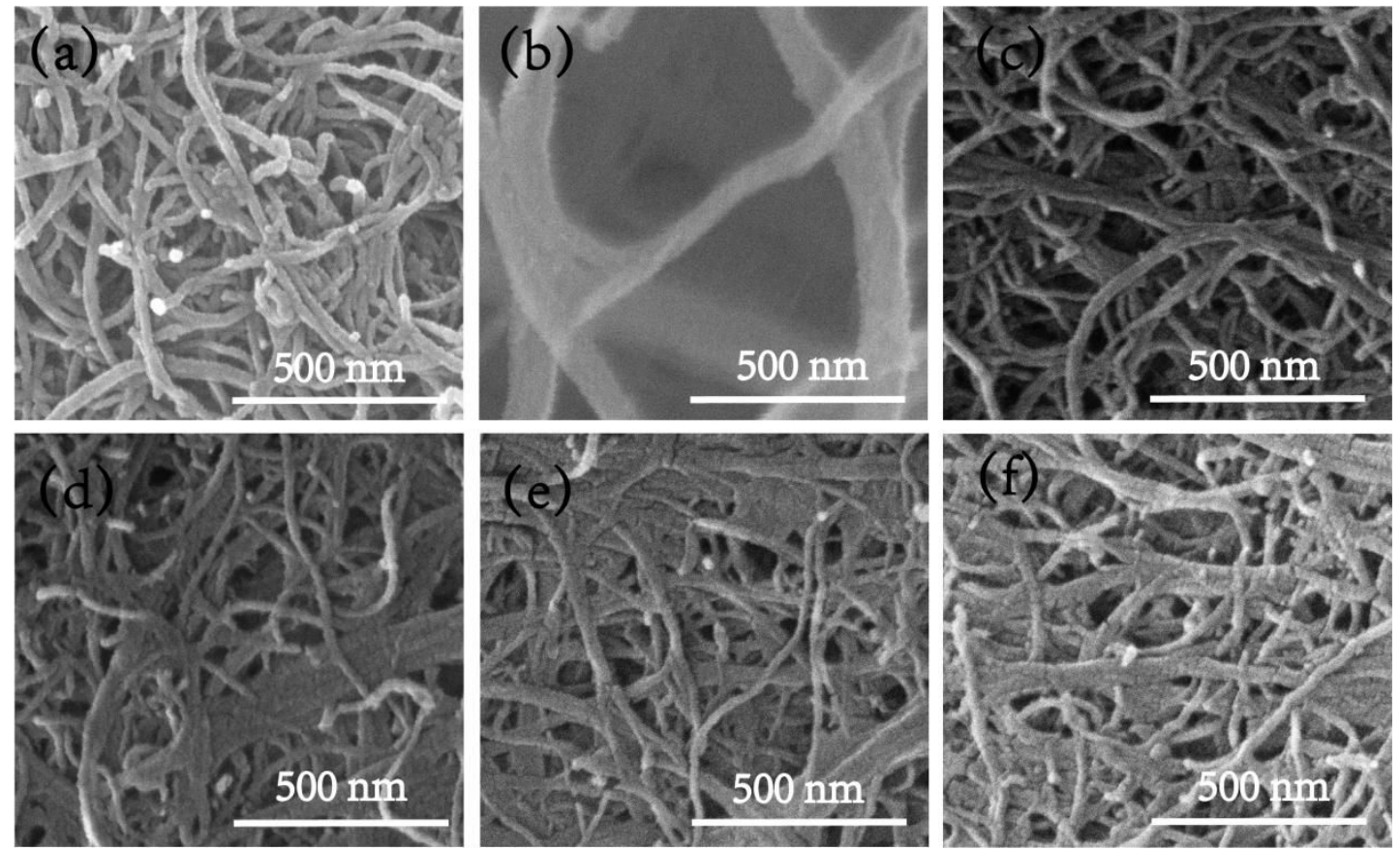

Fig. S1. SEM image of (a) CNT; (b) CNF; (c) CNT/CNF; (d) CNT/Ag $g_{0.5} / \mathrm{CNF}$; (e)

$\mathrm{CNT} / \mathrm{Ag}_{1} / \mathrm{CNF}$; (f) $\mathrm{CNT} / \mathrm{Ag}_{2} / \mathrm{CNF}$. 
The mean diameter ( $\mathrm{D}_{\text {mean }}$ ) of al the silver nanoparticles in the SEM image of $\mathrm{CNT} / \mathrm{Ag}_{1} / \mathrm{CNF}$ were measured using ImageJ to be $6.5 \pm 1.4 \mathrm{~nm}$, as shown in the histogram of Fig S2.

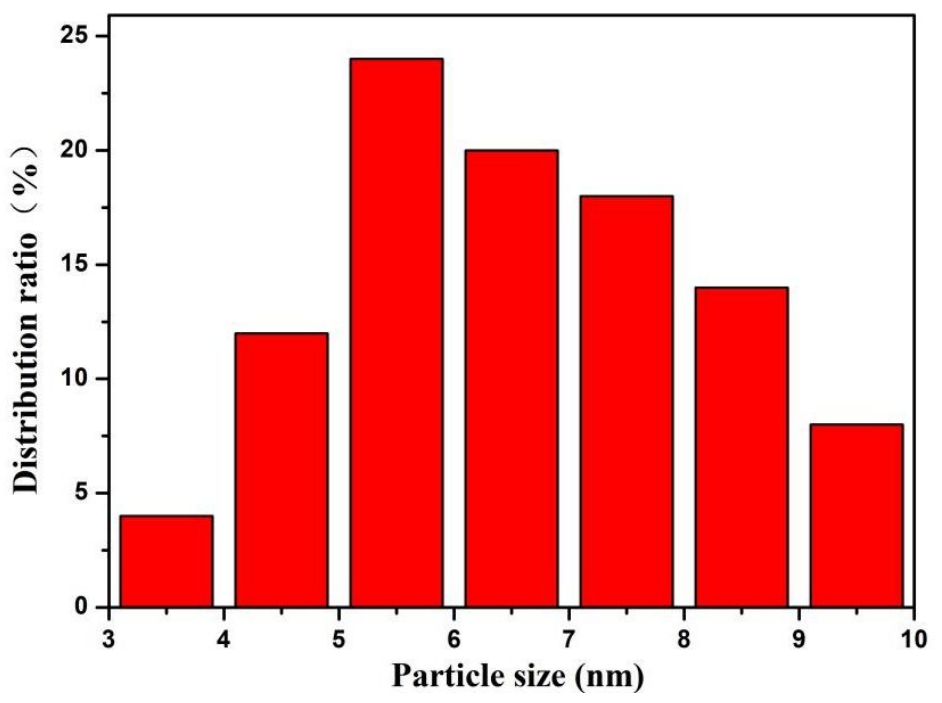

Fig. S2. histogram of silver nanoparticle size 
Fig. S3 presents the $\mathrm{CV}$ curves of $\mathrm{CNT} / \mathrm{Ag}_{1} / \mathrm{CNF}$ in $\mathrm{O}_{2} / \mathrm{N}_{2}$-saturated $0.1 \mathrm{M} \mathrm{KOH}$. Two distinct anodic peaks (A1 and A2) and one cathodic peak (R1) are observed in both $\mathrm{O}_{2}$ and $\mathrm{N}_{2}$-saturated CV curves at $1.24 \mathrm{~V}(\mathrm{~A} 1), 1.33 \mathrm{~V}$ (A2) and $1.08 \mathrm{~V}$ (R1) (vs. RHE). Jinger et al. [1] reported the peak of $\mathrm{A} 1$ is from the formation of the inner hydrous oxide layers of $\mathrm{AgOH}$ and $\mathrm{A} 2$ from the more compact outer layers of $\mathrm{Ag}_{2} \mathrm{O}$. The peak of $\mathrm{R} 1$ is from the reduction of $\mathrm{Ag}_{2} \mathrm{O}$ to Ag. In Fig. S3, one strong reduction peak at $0.77 \mathrm{~V}$ (vs. RHE) is observed in the $\mathrm{O}_{2}$-saturated CV curve. Nevertheless, no such a peak exists in the $\mathrm{N}_{2}$ saturated $\mathrm{KOH}$ electrolyte. Such a peak is termed as oxygen reduction peaks (ORP) in the present work. The ORP indicates that the AgNPs can effectively accelerate the rate of transferring $\mathrm{O}_{2}$ molecules to $\mathrm{HO}_{2}{ }^{-}$ions and enhance the corresponding ORR performance greatly.

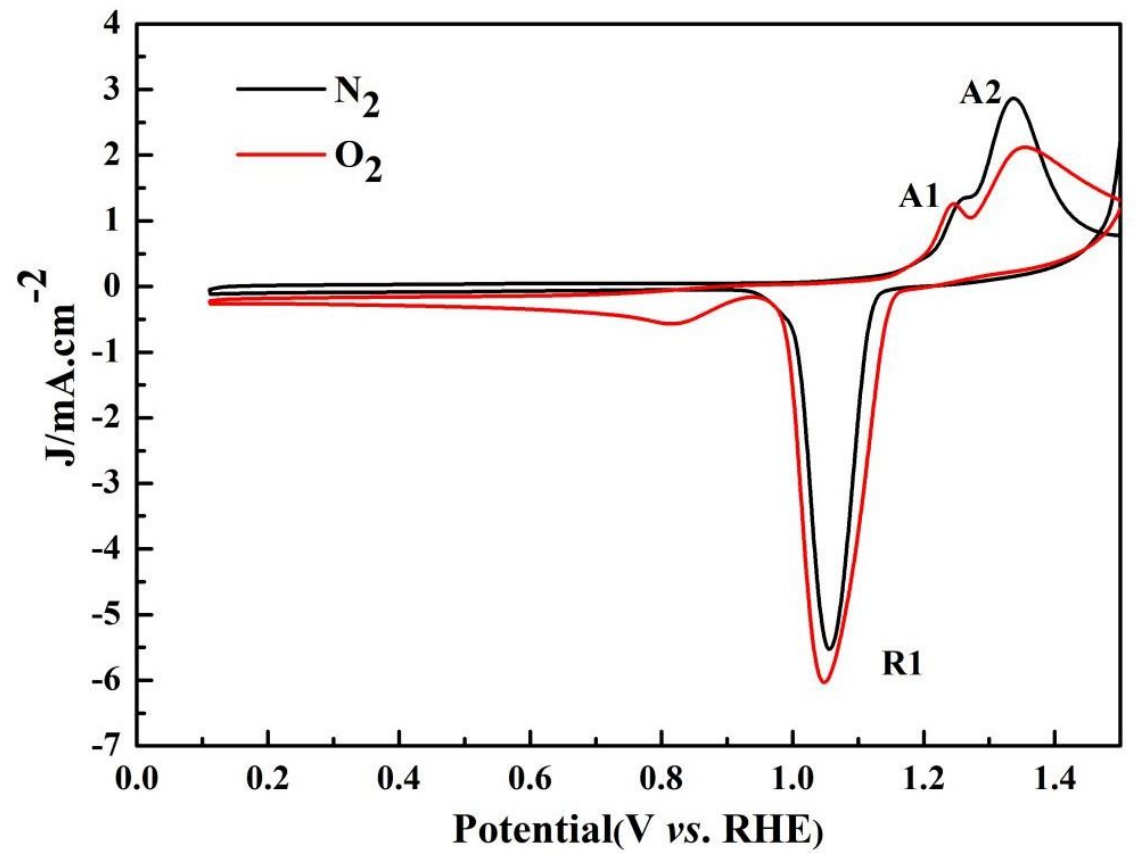

Fig. S3. $\mathrm{CV}$ curves of $\mathrm{CNT} / \mathrm{Ag}_{1} / \mathrm{CNF}$ in $\mathrm{N}_{2} / \mathrm{O}_{2}$ - saturated $0.1 \mathrm{M} \mathrm{KOH}$; 


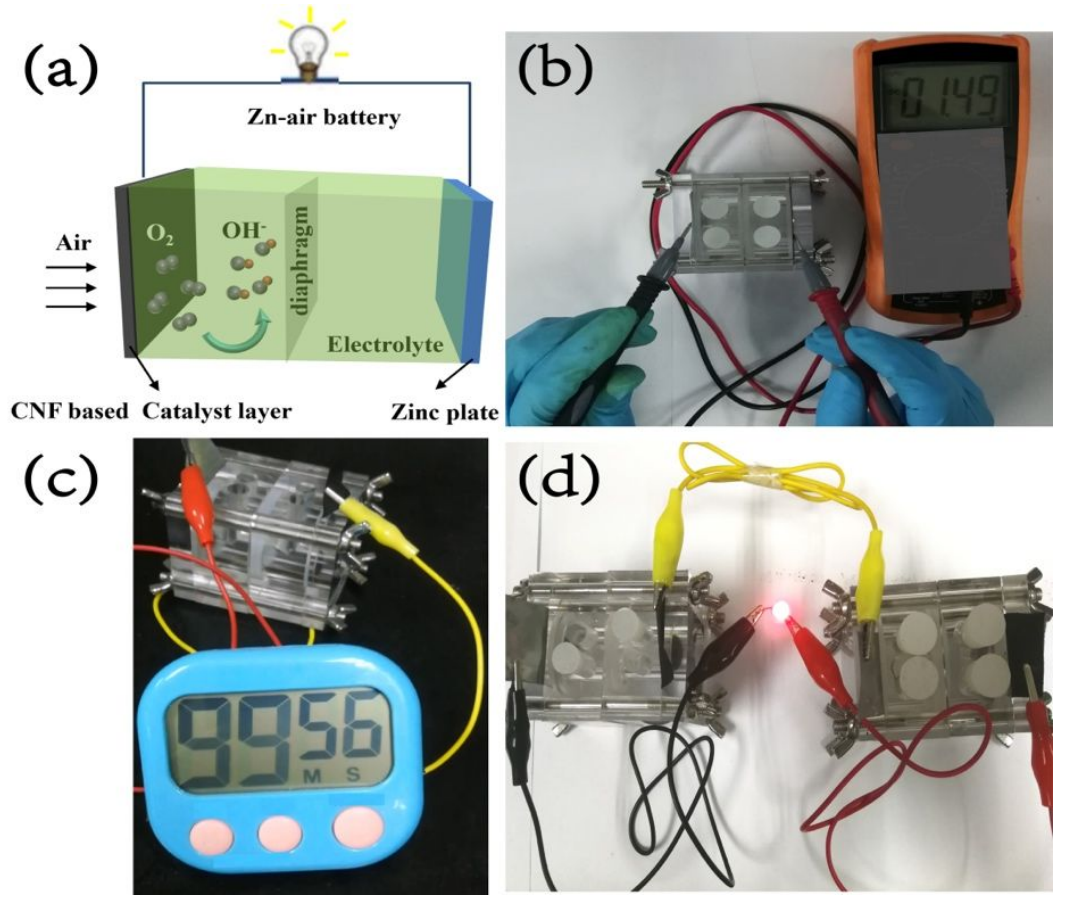

Fig. S4 (a) Schematic diagram of the LZAB configuration with CNF based catalyst as the air cathode; photographs to show: (b) the open circuit voltage of a home-made LZAB; (c) a stopwatch powered by the LZAB for a long time; and (d) a LED light (2V) powered by two home-made CNT/Ag $/ \mathrm{CNF}$ loaded LZABs in series.

\section{(a)}

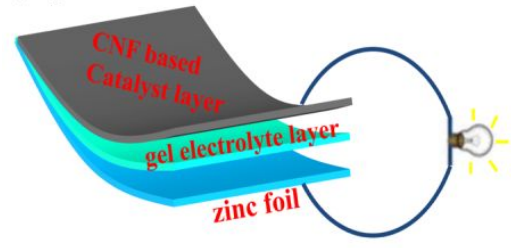

(b)

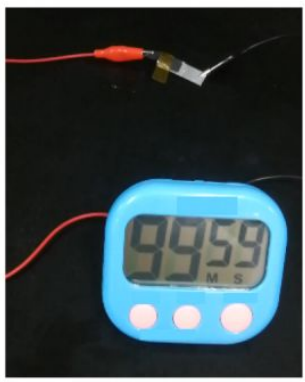

(c)

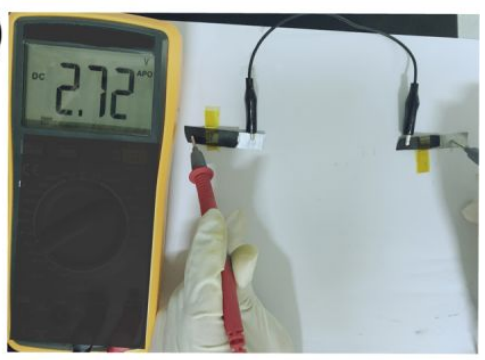

(d)

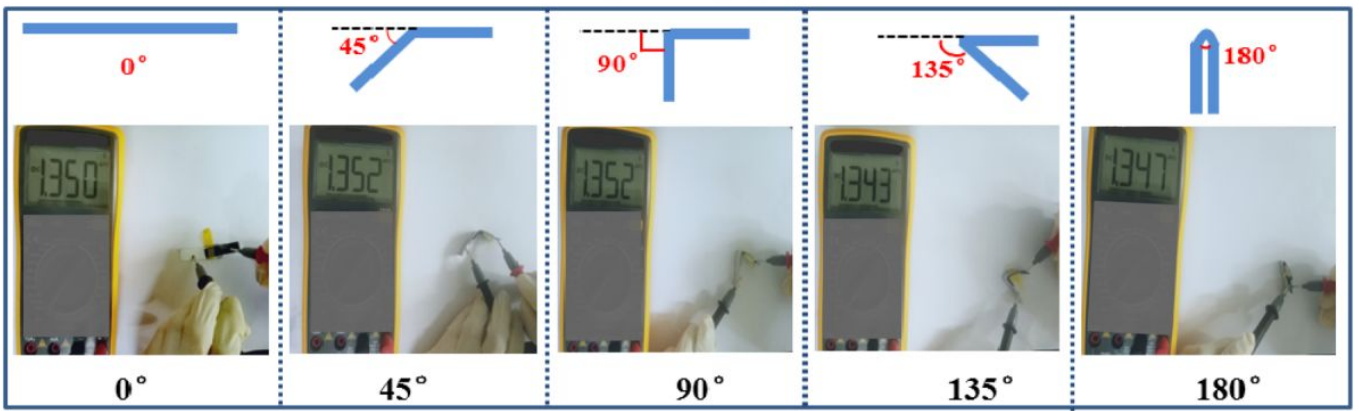

Fig. S5 (a) Schematic illustration of the all-solid-state flexible ZAB configuration; photographs to show (b) a stopwatch powered by a FZAB, (c) the open-circuit voltage of two FZABs in series and (d) the stability of open-circuit voltage of the as-prepared FZAB when bended at various angles of $0,45,90,135$ and $180^{\circ}$ 
Table S1 Chemical reagent parameters for various prepared samples

\begin{tabular}{ccc}
\hline Sample name & $\begin{array}{c}\text { Mass ratio of } \\
\mathrm{CNT} / \mathrm{CNF} / \mathrm{AgNO}_{3}\end{array}$ & $\begin{array}{c}\text { Molar ratio of } \\
\mathrm{AgNO}_{3} / \mathrm{NaBH}_{4}\end{array}$ \\
\hline $\mathrm{CNT} / \mathrm{CNF}$ & $1: 1: 0$ & $1: 10$ \\
$\mathrm{CNT} / \mathrm{Ag}_{0.5} / \mathrm{CNF}$ & $1: 1: 0.5$ & $1: 10$ \\
$\mathrm{CNT} / \mathrm{Ag}_{1} / \mathrm{CNF}$ & $1: 1: 1$ & $1: 10$ \\
$\mathrm{CNT} / \mathrm{Ag}_{2} / \mathrm{CNF}$ & $1: 1: 2$ & $1: 10$ \\
\hline
\end{tabular}

Table S2 Comparison of ORR in CNT/ $\mathrm{Ag}_{\mathrm{x}} / \mathrm{CNF}$ and other electrocatalysts in $0.1 \mathrm{M} \mathrm{KOH}$

\begin{tabular}{lcccc}
\hline \multicolumn{1}{c}{ Catalyst } & $\begin{array}{c}\text { Onset potential } \\
(\text { V vs. RHE })\end{array}$ & $\begin{array}{c}\text { Half potential } \\
(\text { V vs. RHE })\end{array}$ & $\begin{array}{c}\text { Limiting Current } \\
\text { density }\left(\mathrm{mA} \cdot \mathrm{cm}^{-2}\right)\end{array}$ & Reference \\
\hline $\mathrm{CNF}$ & \multicolumn{3}{c}{ No electrocatalytic properties } & This work \\
$\mathrm{CNT} / \mathrm{CNF}$ & 0.76 & 0.560 & -3.3 & This work \\
$\mathrm{CNT} / \mathrm{Ag}_{0.5} / \mathrm{CNF}$ & 0.861 & 0.722 & -4.5 & This work \\
$\mathrm{CNT} / \mathrm{Ag}_{1} / \mathrm{CNF}$ & 0.874 & 0.724 & -5.1 & This work \\
$\mathrm{CNT} / \mathrm{Ag}_{2} / \mathrm{CNF}$ & 0.872 & 0.707 & -4.8 & This work \\
$\mathrm{Pt} / \mathrm{C}$ & 0.98 & 0.874 & -5.8 & This work \\
$\mathrm{Ag} / \mathrm{N}-\mathrm{RGO}$ & 0.880 & 0.76 & -5.3 & {$[2]$} \\
$\mathrm{AMO} / \mathrm{CNTs}-10 \% \mathrm{Ag}$ & 1.07 & 0.69 & -5.1 & {$[3]$} \\
$\mathrm{Ag} / \mathrm{OMMC}$ & 0.92 & 0.79 & -5.6 & {$[4]$} \\
\hline
\end{tabular}

\section{References}

1. Linge, J. M.; Erikson, H.; Kasikov, A.; Rähn, M.; Sammelselg, V.; Tammeveski, K., Oxygen reduction reaction on thin-film Ag electrodes in alkaline solution. Electrochimica Acta 2019, 325.

2. Li, S.; Miao, H.; Xu, Q.; Xue, Y.; Sun, S.; Wang, Q.; Liu, Z., Silver nanoparticles supported on a nitrogen-doped graphene aerogel composite catalyst for an oxygen reduction reaction in aluminum air batteries. RSC Advances 2016, 6 (101), 99179-99183.

3. Sun, H.; Hu, Z.; Yao, C.; Yu, J.; Du, Z., Silver Doped Amorphous MnO2 as Electrocatalysts for Oxygen Reduction Reaction in Al-Air Battery. J. Electrochem. Soc. 2020, 167 (8), 080539.

4.. $\quad$ Dong, J.; Sun, T.; Li, S.; Shan, N.; Chen, J.; Yan, Y.; Xu, L., 3D ordered macro- 
/mesoporous carbon supported Ag nanoparticles for efficient electrocatalytic oxygen reduction reaction. J. Colloid Interface Sci. 2019, 554, 177-182. 